Portland State University

PDXScholar

$1-1-1989$

\title{
Pressure dependence of ozone interference in the laser fluorescence measurement of $\mathrm{OH}$ in the atmosphere [comment]
}

Thomas M. Hard

Portland State University

C. Y. Chan

A. A. Mehrabzadeh

Robert J. O'Brien

Portland State University

Follow this and additional works at: https://pdxscholar.library.pdx.edu/chem_fac

Part of the Physics Commons

Let us know how access to this document benefits you.

\section{Citation Details}

Hard, T. M., Chan, C. Y., Mehrabzadeh, A. A., \& O'Brien, R. J. (1989). Pressure dependence of ozone interference in the laser fluorescence measurement of $\mathrm{OH}$ in the atmosphere [comment]. Applied Optics, 28(1), 26-27.

This Article is brought to you for free and open access. It has been accepted for inclusion in Chemistry Faculty Publications and Presentations by an authorized administrator of PDXScholar. Please contact us if we can make this document more accessible: pdxscholar@pdx.edu. 
Table I. Comparison Between Computed Transmittances

\begin{tabular}{crcccc}
\hline \multicolumn{1}{c}{$g$} & \multicolumn{1}{c}{$\tau$} & GLQM & $\epsilon=2$ & $\epsilon=\sqrt{ } 3$ & AFFM \\
\hline \multirow{3}{*}{0.000} & 0.5 & 0.7975 & 0.7978 & 0.7983 & 0.7974 \\
& 8.0 & 0.1782 & 0.1666 & 0.1723 & 0.1806 \\
& 16.0 & 0.0964 & 0.0882 & 0.0920 & 0.0974 \\
0.750 & 0.5 & 0.9627 & 0.9621 & 0.9636 & 0.9621 \\
& 8.0 & 0.4936 & 0.5162 & 0.5452 & 0.5000 \\
& 16.0 & 0.3120 & 0.3404 & 0.3683 & 0.3212 \\
0.875 & 0.5 & 0.9839 & 0.9827 & 0.9834 & 0.9840 \\
& 8.0 & 0.6919 & 0.7003 & 0.7261 & 0.6894 \\
& 16.0 & 0.4945 & 0.5307 & 0.5632 & 0.5041 \\
\hline
\end{tabular}

The problem of taking absorption into account to specify the ACP will be considered further.

This work was supported by the National Natural Science Foundation of China.

\section{References}

1. B. Maheu, J. N. Letoulouzan, and G. Gouesbet, "Four-Flux Models to Solve the Scattering Transfer Equation in Terms of LorenzMie Parameters," Appl. Opt. 23, 3353 (1984).

2. B. Maheu and G. Gouesbet, "Four-Flux Models to Solve the Scattering Transfer Equation: Special Cases," Appl. Opt. 25, 1122 (1986).

3. G. Kortum, Reflectance Spectroscopy (Springer-Verlag, New York, 1969).

4. A. Ashimaru, Wave Propagation and Scattering in Random Medium (Academic, New York, 1978).

5. D. W. Marquardt, "An Algorithm for Least-Squares Estimation of Nonlinear Parameters," J. Soc. Ind. Appl. Math. 11, 431 (1963).

6. H. C. van de Hulst, Multiple Light Scattering (Academic, New York, 1980).

\section{Pressure dependence of ozone interference in the laser fluorescence measurement of $\mathrm{OH}$ in the atmosphere: comment}

\section{T. M. Hard, C. Y. Chan, A. A. Mehrabzadeh, and Robert J.} O'Brien

Portland State University, Portland, Oregon 97207-0751. Received 11 January 1988.

0003-6925/89/010026-02\$02.00/0.

(C) 1989 Optical Society of America.

Shirinzadeh et al. ${ }^{1}$ have treated the production of spurious concentrations of hydroxyl during laser excited fluorescence measurement of this radical's atmospheric concentration. The interfering $\mathrm{OH}$ results from ozone photolysis, producing $O\left({ }^{1} \mathrm{D}\right)$, which reacts with water vapor to produce $\mathrm{OH}$, which is detected during the same laser pulse. These authors ${ }^{1}$ show that the time-averaged $\mathrm{OH}$ concentration produced during a laser pulse of width $\Delta t$ is given by

$$
[\mathrm{OH}]_{i}=(1 / A) k\left[\mathrm{H}_{2} \mathrm{O}\right]\left[\mathrm{O}_{3}\right] E A_{c}^{\prime} F(A \Delta t) .
$$

Here $A$ is the first-order rate coefficient for the quenching of $\mathrm{O}\left({ }^{1} \mathrm{D}\right)$ by air, $\sigma$ is the absorption cross section of $\mathrm{O}_{3}$ in $\mathrm{cm}^{2}, k$ is the second-order rate coefficient for reaction of $\mathrm{O}\left({ }^{1} \mathrm{D}\right)$ with $\mathrm{H}_{2} \mathrm{O}, E$ is the pulsed photon flux through the sample in photons $\mathrm{cm}^{-2}, A_{c}^{\prime}$ is the fraction of the $\mathrm{OH}$ [produced by the $\mathrm{O}\left({ }^{1} \mathrm{D}\right)+\mathrm{H}_{2} \mathrm{O}$ reaction] that resides initially in the rotational/ vibrational level probed by the same laser pulse and $F(A \Delta t)$ $=1-(2 / A \Delta t)\{1-(1 / A \Delta t)[1-\exp (-A \Delta t)]\}$. The principal assumption implied by Eq. (1) is that rotational relaxation of $\mathrm{OH}$ during the pulse does not contribute to the observed concentration over the pressure range of interest (from 2 to 760 Torr). This assumption is questionable at the highest pressures in this range; yet it appears to be supported by experimental data (1) on the pressure dependence of Eq. 1, at least within experimental uncertainty.

In $\mathrm{OH}$ detectors that lower the sample pressure before laser excitation, ${ }^{1-3}$ the consequent $\mathrm{OH}$ density changes are compensated by changes in fluorescence efficiency, ${ }^{4}$ down to a lower limiting pressure of $<2$ Torr. If Shirinzadeh et al.'s assumption $^{1}$ of zero chemical loss of ambient $\mathrm{OH}$ during expansion is correct, the equivalent ambient $\mathrm{OH}$ concentration that corresponds to the absolute interference in Eq. (1) is $[\mathrm{OH}]_{i a}=\left(P_{a} / P_{d}\right)[\mathrm{OH}]_{i}$ where $P_{a}$ and $P_{d}$ are the ambient and detection pressures, respectively.

Although we commend Shirinzadeh et al. on their development and experimental verification of Eq. (1) we must point out three errors which caused them to overpredict spurious $\mathrm{HO}$ in our published data ${ }^{2,3}$ more than 3 orders of magnitude.

The most serious disagreement concerns the net interference obtained from the ozone/water mechanism when a hydrocarbon is added periodically to the sample flow to measure the total background from all causes. Shirinzadeh et al., who did no experiments with hydrocarbon modulation, state correctly that the presence of the hydrocarbon increases the removal rate of $\mathrm{O}\left({ }^{1} \mathrm{D}\right)$. This in turn reduces the production of $\mathrm{OH}$, thereby reducing the gross photolytic background represented by Eq. (1), resulting in a false positive $\mathrm{OH}$ signal. This signal $[\mathrm{OH}]_{\text {spur }}$ is equal to the difference between Eq. (1) as written and Eq. (1) with substitution of $A+k_{\mathrm{H}}$ for $A$. Here $k_{\mathrm{H}}$ is the first-order $\mathrm{O}\left({ }^{1} \mathrm{D}\right)$ removal rate coefficient due to the hydrocarbon concentration alone. Algebraic manipulation yields

$$
[\mathrm{OH}]_{\mathrm{spur}}=[\mathrm{OH}]_{i}\left[1-\frac{F\left(\left[A+k_{\mathrm{H}}\right] \Delta t\right)}{A+k_{\mathrm{H}}} \cdot \frac{A}{F(A \Delta t)}\right] \text {. }
$$

The two ratios of the form $F / A$ in Eq. (2) can be evaluated by approximating the exponential within $F$ by a power series. If $A \Delta t \ll 1$ and $k_{\mathrm{H}} / A \ll 1$, further steps give

$$
[\mathrm{OH}]_{\mathrm{spur}}=[\mathrm{OH}]_{i}\left(k_{\mathrm{H}} \Delta t / 4\right) \text {. }
$$

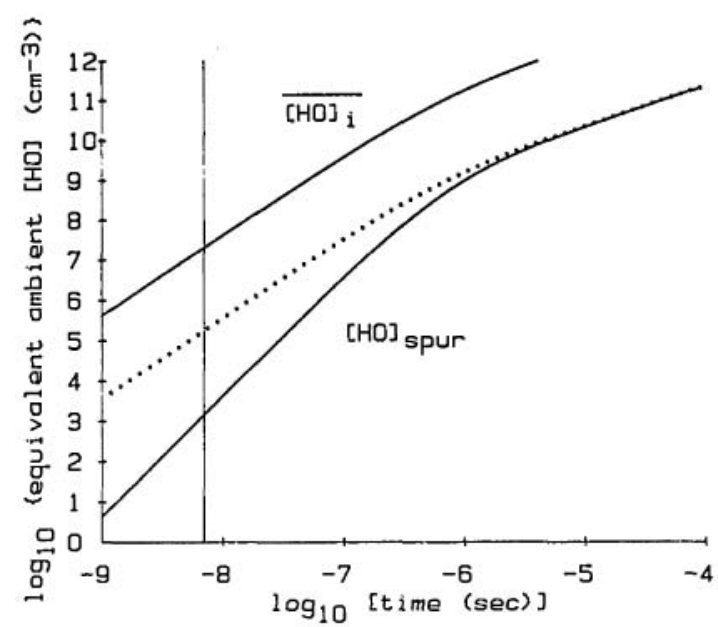

Fig. 1. Log-log plot of predicted gross $[\mathrm{OH}]_{i}$ and net $\left([\mathrm{OH}]_{\text {spur }}\right)$ ozone/water signals vs time. Conditions: air sample containing 200-ppb $\mathrm{O}_{3}$ and 20-Torr $\mathrm{H}_{2} \mathrm{O}$, expanded from 760 to 4 Torr, singlepass excitation at $282 \mathrm{~nm}$ at a constant rate of $1 \mathrm{~mJ} / 7 \mathrm{~ns}$ with beam diameter $0.4 \mathrm{~cm}$, modulation by $460-\mathrm{ppm}$ isobutane, rate coefficient $k_{\mathrm{O}^{1} \mathrm{D}+\mathrm{iBu}}=6.6 \times 10^{-10} \mathrm{~mol}^{-1} \mathrm{~cm}^{3} \mathrm{~s}^{-1}$. Vertical line indicates actual laser pulse width; extension to shorter and longer illumination times illustrates asymptotic behavior of Eq. (2). Dotted curve explained In text. 
Equation (2a) is the low-pressure limit corresponding to the conditions under which chemical modulation has been used. ${ }^{2,3}$ At 4 Torr, $A=4.4 \times 10^{6} \mathrm{~s}^{-1}$; with $\Delta t=7 \mathrm{~ns}, A \Delta t=$ 0.03 . The added isobutane concentration of $460 \mathrm{ppm}$ and an $\mathrm{O}\left({ }^{1} \mathrm{D}\right)+$ isobutane rate coefficient of $6.6 \times 10^{-10} \mathrm{~mol}^{-1} \mathrm{~cm}^{3}$ $\mathrm{s}^{-1}$ give $k_{\mathrm{H}}=4 \times 10^{4} \mathrm{~s}^{-1}$. The resulting fractional modulation $[\mathrm{OH}]_{\text {spur }} /[\mathrm{OH}]_{i}$ is $k_{\mathrm{H}} \Delta t / 4=7 \times 10^{-5}$. That is, only $7 \times$ $10^{-5}$ of the gross photolytic HO shows up as a net positive offset in this mechanism.

At higher pressures, $A \Delta t \gg 1$, and Eq. (2) approaches

$$
[\mathrm{OH}]_{\mathrm{spur}}=[\mathrm{OH}]_{i}\left(k_{H} / A\right),
$$

which is identical to Eq. (6) of Ref. 1. When Eq. (2b) is mistakenly applied to the low-pressure case, $[\mathrm{OH}]_{\text {spur }}$ is overpredicted by the factor $\left(k_{H} / A\right) /\left(k_{H} \Delta t / 4\right)=4 / A \Delta t$. For $A \Delta t$ $=0.03$, appropriate to Refs. 2 and 3, Shirinzadeh et al. used Eq. (2b), overpredicting the fractional modulation by a factor of more than 100.

Figure 1 displays the time behavior of Eqs. (1), (2), and (2b), assuming continued illumination of the sample at the same rate as the average during laser pulse. The top curve is the gross ozone/water background predicted by Eq. (1). The bottom curve is the net chemical modulation given by Eq. (2), which is adequately approximated by Eq. (2a) at short pulse widths and low pressures. The dotted curve is obtained by arbitrarily setting $F\left(\left[A+k_{\mathrm{H}}\right] \Delta t\right)=F(A \Delta t)$ in Eq. (2), equivalent to the erroneous application of Eq. (2b) to all pressures. The error in the misuse of Eq. (2b) is represented by the displacement of the dotted curve from the solid curve $\left([\mathrm{OH}]_{\text {spur }}\right)$ at the vertical line indicating our laser pulse width.

The right-hand portions of these curves are displayed only to exhibit the asymptotic behavior of Eqs. (1) and (2). In reality, one expects increasing contributions of rotational and vibrational relaxation of $\mathrm{OH}$ after $10^{-6} \mathrm{~s}$. After $10^{-4} \mathrm{~s}$, chemical and bulk-transport losses of $\mathrm{OH}$ become significant. Neglecting these complications, Eqs. (1) and (2) are linear in $t$ in the steady-growth limit. At times shorter than $1 / A=220 \mathrm{~ns}$ the graph shows $t^{2}$ dependence ${ }^{5}$ for $[\mathrm{OH}]_{i}$ and $t^{3}$ dependence for $[\mathrm{OH}]_{\text {spur, }}$, agreeing with the leading terms of their respective series approximations. Both variables were defined as averages from $t=0$ to $t$. Although not plotted in Fig. 1, the corresponding instantaneous kinetic variables at $t$ are larger than the averages by nearly constant factors: 2 in the linear-growth region, 3 in the $t^{2}$ region of $[\mathrm{OH}]_{i}$ and 4 in the $t^{3}$ region of the net yield from chemical modulation.

Our second objection concerns the theoretical prediction of the gross ozone-water background for the ambient $\mathrm{OH}$ measurements reported in Ref. 2, using Eq. (1). Reference 2 did not supply the actual beam diameter $(0.4 \mathrm{~cm})$ in the detection zone or the laser pulse width $(7 \mathrm{~ns})$. These corrections reduce the predicted gross background by a factor of 9 .

Together, the above corrections to the gross and net photolytic signals lower $[\mathrm{OH}]_{\text {spur }}$ from that predicted for single laser beam pass excitation by Shirinzadeh et al. ${ }^{1}$ by a factor of more than $10^{3}$.

Our third comment concerns $\mathrm{HO}$ production by one channel of the reaction of $\mathrm{O}\left({ }^{1} \mathrm{D}\right)$ with the hydrocarbon, ignored by Shirinzadeh et al. Such production has been observed for other small alkanes ${ }^{7}$ and would be expected for isobutane. Since this production of detectable $\mathrm{OH}$ increases the signal in the background channel, it is a false negative $\mathrm{OH}$ signal. This negative contribution, as well as the effect of increased quenching of spurious $\mathrm{OH}\left(A^{2} \Sigma\right)$ by isobutane and the increased net interference due to overlap of beams within our White cell, will be treated elsewhere. ${ }^{8}$ Using isobutane as the modulating hydrocarbon, we find experimentally that the net result of $\mathrm{O}\left({ }^{1} \mathrm{D}\right)$ modulation, $\mathrm{OH}\left(A^{2} \Sigma\right)$ quenching, and additional $\mathrm{OH}$ production is a negative interference for all atmospheric $\mathrm{H}_{2} \mathrm{O}$ concentrations.

As a consequence, the presence of spurious $\mathrm{OH}$ is readily detected by nighttime measurements using chemical modulation. If ozone is present, such measurements produce a negative offset recognizable since nighttime $\mathrm{OH}$ concentrations are quite low or zero. We routinely perform $24-\mathrm{h}$ experiments and have in fact reported a nighttime offset of 2 $\times 10^{5} \mathrm{HO} \mathrm{cm}-3 .{ }^{2}$ At the time this offset was attributed to possible fluorescence of impurities in the isobutane modulating reagent, an offset which is constant and subtractable. Although the ozone offset is small, it is not constant and should be minimized or accurately measured. This topic will be treated elsewhere. ${ }^{8}$

\section{References}

1. B. Shirinzadeh, D. Q. Deng, and C. C. Wang, "Pressure Dependence of Ozone Interference in the Laser Fluorescence Measurement of OH in the Atmosphere," Appl. Opt. 26, 2102 (1987).

2. T. M. Hard, R. J. O'Brien, C. Y. Chan, and A. A. Mehrabzadeh, "Diurnal Cycle of Tropospheric OH," Nature London 322, 617 (1986).

3. T. M. Hard, R. J. O'Brien, C. Y. Chan, and A. A. Mehrabzadeh, "Trophospheric Free Radical Determination by FAGE," Environ. Sci. Technol. 18, 768 (1984).

4. T. M. Hard, R. J. O'Brien, T. B. Cook, and G. A. Tsongas, "Interference Suppression in HO Fluorescence Detection," Appl. Opt. 18, 3217 (1979).

5. The $t^{2}$ dependence of $[\mathrm{OH}]_{i}$ found here is not inconsistent with the $t^{3}$ dependence found ${ }^{6}$ in the case where collisional relaxation of excited $\mathrm{OH}$ was assumed to be the sole path to the detected rotational state.

6. M. Hanabusa, C. C. Wang, S. Japar, D. K. Killinger, and W. Fisher, "Pulsewidth Dependence of Ozone Interference in the Laser Fluorescence Measurement of $\mathrm{OH}$ in the Atmosphere," J. Chem. Phys. 66, 2118 (1977).

7. A. C. Luntz, "Chemical Dynamics of the Reactions of $\mathrm{O}\left({ }^{1} \mathrm{D}_{2}\right)$ with Saturated Hydrocarbons," J. Chem. Phys. 73, 1143 (1980).

8. T. M. Hard, C. C. Chan, A. A. Mehrabzadeh, and R. J. O'Brien, in preparation.

\section{Phase-stepping interferometry with laser diodes: effect of changes in laser power with output wavelength}

\section{P. Hariharan}

CSIRO Division of Applied Physics, Sydney, NSW 2070, Australia.

Received 23 August 1988.

0003-6935/89/010027-03\$02.00/0.

(C) 1989 Optical Society of America.

In phase-stepping interferometry the mean phase difference between the two interfering beams is varied in equal steps of known magnitude and measurements are made of the corresponding values of the intensity at a network of points covering the interference pattern. Three or more of these intensity values can then be used to calculate the original phase difference between the interfering beams at each of these points. ${ }^{1}$

The most common method of introducing the phase steps is by mounting one of the mirrors of the interferometer on a piezoelectric transducer (PZT) and applying suitable voltages to the PZT. However, this method is not easy to apply with large and heavy mirrors. An alternative method which 